Broad minded on narrow spikes

\section{Robert Meech}

IT Is natural to suppose that the longer and larger the action potential that arrives at a chemical synapse, the greater the rise in intracellular $\mathrm{Ca}^{2+}$ and the more neurotransmitter that it causes to be released. Just such a mechanism may contribute to the analgesic action of opioids in vertebrates ' and to behavioural sensitization in Aplysia $^{2}$. But on page 636 of this issue ${ }^{3}$, A. N. Spencer, J. Przysiezniak and J. Acosta-Urquidi report that at the neuromuscular junction of the jellyfish Polyorchis penicillatus precisely the reverse is true: short action potentials produce a larger postsynaptic event than longer ones. This is not a specialization of simple systems like jellyfish, for the explanation is a general one with implications for nervous systems as complex as those of the insects or mammals.

In 1967, Katz and Miledi ${ }^{4}$ used squid axon giant synapses treated with tetrodotoxin to show that transmitter release depends on the amplitude and duration of the depolarization at the presynaptic terminal. In depolarized squid axons, calcium activation develops so slowly that the rise in internal $\mathrm{Ca}^{2+}$ follows much the same course as the increase in potassium conductance $^{5}$. Hence, when Llinás, Steinberg and Walton ${ }^{6}$ reconstructed the events during normal synaptic transmission, they showed that the calcium responsible for transmitter release enters the terminal during the repolarizing phase of the action potential. Accordingly, the slower the rate of repolarization, the greater the calcium influx at the presynaptic terminal and the larger the associated postsynaptic response. This has been confirmed experimentally in vertebrates by Lin and $\mathrm{Faber}^{7}$

using synapses on the Mauthner cell of the goldfish.

Prolonged action potentials are not always associated with a greater rise in intracellular $\mathrm{Ca}^{2+}$, however. In molluscan neurons, successive impulses in a train of action potentials grow in duration as a result of the cumulative depression of voltage-dependent potassium currents ${ }^{8}$. Use of the calcium-sensitive dye arsenazo III injected into the giant neurons of Aplysia has shown that the action potentials may broaden by as much as 50 per cent with no apparent change in calcium influx". This is because the pronounced shoulder on the falling phase of each of the prolonged action potentials is near $0 \mathrm{mV}$. Most calcium channels are closed at this voltage in these cells and so the prolongation has little effect on the change in intracellular $\mathrm{Ca}^{2+}$ measured by arsenazo.

Compared with Aplysia, the position of the calcium activation curve in squid ${ }^{10}$ and Polyorchis ${ }^{3}$ is shifted along the voltage axis so that there is a substantial inward current at $0 \mathrm{mV}$; this is illustrated for the Polyorchis motor neuron, ( $a$ in the figure) which shows a maximum inward current at about $+10 \mathrm{mV}$. As a result, prolongation is most effective with relatively low amplitude action potentials. This is because the driving force on the calcium ion decreases as the membrane potential approaches the calcium equilibrium potential and, although the calcium channels are open, calcium entry becomes smaller not greater. With a large amplitude action potential it is not until the membrane begins to repolarize that the driving force becomes sufficient to promote calcium entry.

The Polyorchis preparation differs from


a. Step changes in membrane potential at the presynaptic terminal produce a transient inward calcium current across the terminal membrane. In this representation of data from Polyorchis motor neurons ${ }^{3}$ the peak of the inward calcium current is shown on the horizontal axis with the membrane potential plotted vertically. The range of membrane potentials at which the calcium current is greater than 75 per cent of maximum lies between the dotted lines. In Aplysia neurons the relationship between inward calcium current and membrane potential is displaced to more positive voltages (upward in the figure); in squid axons the relationship is displaced downward. b,c Intracellularly recorded action potentials from Polyorchis ${ }^{3}$ showing that the calcium influx is near maximum for almost the entire time that the smaller shorter action potential $(b)$ overshoots $0 \mathrm{mV}$, whereas the longer action potential $(c)$ enters this range only after a considerable delay. the squid synapse in that its action potential is sufficiently prolonged for calcium channel inactivation to become significant. In Polyorchis, the entire overshoot of the shorter action potential is associated with a near maximum inward calcium current ( $b$ in the figure) but the peak of the longer action potential is out of this range ( $c$ in the figure) and so there is relatively little calcium entry until the later stages of repolarization. Spencer et al. suggest that during the early stages of the action potential many calcium channels are inactivated and become unavailable. As a result, calcium enters the cytoplasm at a low rate and much of it is sequestered by cytoplasmic calcium buffers. With entry and sequestration rates nearly the same, there would be little change in $\mathrm{Ca}^{2+}$ and little release of transmitter, just as Spencer et al. observe.

Although it seems that the basic mechanism of synaptic transmission may be universal the performance of each synapse, whether from goldfish, squid or jellyfish, depends on the shape of the presynaptic action potential, the voltage dependence and kinetics of the presynaptic calcium channel, and the kinetics of cellular regulation. More preparations are needed to evaluate the influence of these different factors. The Polyorchis preparation developed by Spencer and colleagues has the advantage of good electrical access to the synaptic sites and so further dissection of the presynaptic currents may be possible. But it is just one such preparation identified in jellyfish. Others are the neuromuscular synapse of Aglantha", with its dual system of action potentials, and the bidirectional synapse of Cyanea ${ }^{12}$. Researchers with patrons farsighted enough to support these ventures into the realms of the jellyfish have returned with great bounties. The advantages of using the simple nervous system of the jellyfish for experimentation are more than just travellers' tales.

Robert Meech is the Wellcome Trust Senior Lecturer in Physiology at the University of Bristol Medical School, Bristol BS8 1TD, UK.

1. Mudge, A Leeman, S. \& Fishbach, G.D. Proc natn Acad. Sci. U.S.A. 76, 526-530 (1979).

2. Klein, M. \& Kandel, E.R. Proc. natn. Acad. Sci. U.S.A. 75, 3512-3516 (1978)

3. Spencer, A.N., Przysiezniak, J. \& Acosta-Urquidi, J Nature 340, 636-638 (1989).

4. Katz, B. \& Miledi, R. J. Physiol., Lond. 192, 407-436 (1967).

5. Baker, P.F., Hodgkin, A.L. \& Ridgeway, E.B. J. Physiol. Lond. 218, 709-755 (1971)

6. Llinás, R., Steinberg, 1.Z. \& Walton, K. Biophys. J. 33, 289-322 (1981)

7. Lin, J-W. \& Faber, D.S. J. Neurosci. 8, 1313-1325 (1988)

8. Aldrich, R.W., Getting, P.A. \& Thompson, S.H. J. Physiol. Lond. 291, 531-544 (1979).

9. Smith, S.J. \& Zucker, R.S. J. Physiol., Lond. 3c0, 167 196 (1980)

10. Augustine, G.J., Charlton, M.P. \& Smith, S.J. J. Phy siol., Lond. 367, 143-162 (1985).

11. Mackie, G.O \& Meech, R.W. Nature 313, 791-793 (1982).

12. Anderson, P.A.V. J. Neurophysiol. 53, 821-835 (1985)

NATURE · VOL $340 \cdot 24$ AUGUST 1989 